\title{
An improved estimation procedure of population mean using bivariate auxiliary information under non-response
}

\author{
Shashi Bhushan ${ }^{a}$, Abhay Pratap Pandey ${ }^{1, b}$ \\ ${ }^{a}$ Department of Mathematics and Statistics, DSMNR University, India \\ ${ }^{b}$ Ramanujan College, University of Delhi, India
}

\begin{abstract}
We propose new classes of estimators of population mean under non-response using bivariate auxiliary information. Some improved regression (or difference) type estimators have been proposed in four different situations of non response along with their properties and the expressions for the bias and mean square errors of the proposed estimators are derived under double (two-stage) sampling scheme. The properties of the suggested class of estimators are studied and it is observed that the proposed estimators performed better when compared to conventional estimators proposed by Singh and Kumar (Journal of Statistical Planning and Inference, 140, 2536-2550, 2010b), Shabbir and Khan (Communications in Statistics - Theory and Methods, 42, 4127-4145, 2013) and Bhushan and Naqvi (Journal of Statistics and Management Systems, 18, 573-602, 2015). A comparative study is also conducted both theoretically as well as empirically in order to support the results.
\end{abstract}

Keywords: auxiliary information, non-response, mean square error

\section{Introduction}

Survey statistician often make use of available auxiliary information to improve the precision of estimates. Situations where the population mean of the auxiliary variable is known and the non-response is present have been dealt with by various authors including Cochran (1977), Rao (1986, 1987), Khare and Srivastava (1993), Singh and Kumar (2008). However, in situations when the population mean of the auxiliary variable is unknown, the sample mean $\bar{x}^{\prime}$ obtained from a large first phase sample of size $n^{\prime}$ drawn from $N$ units by simple random sampling without replacement is used as suggested by Khare and Srivastava (1995, 1997), Okafor and Lee (2000), Tabasum and Khan (2004) and recently by Singh et al. (2010), Singh and Kumar (2010a) among others. It is assumed that all first phase sample units supplied the auxiliary information, then, a second phase sample of size $n\left(n<n^{\prime}\right)$ is drawn from the $n^{\prime}$ by SRSWOR and study variable $y$ is measured on it. At the second phase from the sample of size $n$, let $n_{1}$ units respond and $n_{2}$ units refuse to respond. Now, we use Hansen and Hurwitz (1946) sampling strategy to sub-sample $r$ units from $n_{2}$ non-responding units and enumerated by direct interview so that $r=n_{2} / k, k>1$.

This paper considers the deterministic setup of non-response exactly on the similar lines of Okafor and Lee (2000) and assumes that the whole population (denoted by $\Omega$ ) is divided into two groups, one is that of the responding group of $N_{1}$ units (denoted by $\Omega_{1}$ ) and the other non-responding group of $N_{2}$ units (denoted by $\Omega_{2}$ ). Let the first and second phase samples be denoted by $u$ and $u^{\prime}$ respectively, and let $u_{1}=u \cap \Omega_{1}$ and $u_{2}=u \cap \Omega_{2}$. The sub sample from $u_{2}$ is denoted by $u_{(2)}$.

\footnotetext{
${ }^{1}$ Corresponding author: Ramanujan College, University of Delhi, Kalkaji New Delhi-110019, India.

E-mail: abhaypratap.pandey@gmail.com
}

Published 31 July 2019 / journal homepage: http://csam.or.kr

(c) 2019 The Korean Statistical Society, and Korean International Statistical Society. All rights reserved. 


\section{Existing estimators}

The conventional ratio and regression type estimators for the population mean when there is nonresponse on the study variable as well as on the auxiliary variable are given as

$$
\begin{aligned}
\bar{y}_{R(1)} & =\bar{y}^{*} \frac{\bar{x}^{\prime}}{\bar{x}^{*}}, \\
\bar{y}_{\operatorname{Reg}_{(1)}} & =\bar{y}^{*}+b_{y x}^{*}\left(\bar{x}^{\prime}-\bar{x}^{*}\right)
\end{aligned}
$$

the mean squared error (MSE)'s of these estimators are given by

$$
\begin{aligned}
\operatorname{MSE}\left(\bar{y}_{R(1)}\right) & =\bar{Y}^{2}\left[\left(f_{n}-f_{n}^{\prime}\right)\left\{C_{y}^{2}+\left(1-2 K_{y x}\right) C_{x}^{2}\right\}+g_{n}\left\{C_{y(2)}^{2}+\left(1-2 K_{y x(2)}\right) C_{x(2)}^{2}\right\}+f_{n}^{\prime} C_{y}^{2}\right], \\
\operatorname{MSE}\left(\bar{y}_{\operatorname{Reg}_{(1)}}\right) & =\bar{Y}^{2}\left[\left(f_{n}-f_{n}^{\prime}\right)\left(1-\rho_{y x}^{2}\right) C_{y}^{2}+g_{n}\left\{C_{y(2)}^{2}+K_{y x}\left(K_{y x}-2 K_{y x(2)}\right) C_{x(2)}^{2}\right\}+f_{n}^{\prime} C_{y}^{2}\right]
\end{aligned}
$$

and the conventional ratio and regression type estimators for population mean, when there is a nonresponse on the study variable alone, are given as

$$
\begin{aligned}
\bar{y}_{R(2)} & =\bar{y}^{*} \frac{\bar{x}^{\prime}}{\bar{x}}, \\
\bar{y}_{\operatorname{Reg}_{(2)}} & =\bar{y}^{*}+b_{y x}^{* *}\left(\bar{x}^{\prime}-\bar{x}\right)
\end{aligned}
$$

the MSE's of these estimators are given by

$$
\begin{aligned}
\operatorname{MSE}\left(\bar{y}_{R(2)}\right) & =\bar{Y}^{2}\left[\left(f_{n}-f_{n}^{\prime}\right)\left\{C_{y}^{2}+\left(1-2 K_{y x}\right) C_{x}^{2}\right\}+g_{n} C_{y(2)}^{2}+f_{n}^{\prime} C_{y}^{2}\right], \\
\operatorname{MSE}\left(\bar{y}_{\operatorname{Reg}_{(2)}}\right) & =\bar{Y}^{2}\left[\left(f_{n}-f_{n}^{\prime}\right)\left(1-\rho_{y x}^{2}\right) C_{y}^{2}+g_{n} C_{y(2)}^{2}+f_{n}^{\prime} C_{y}^{2}\right]
\end{aligned}
$$

Singh and Kumar (2010b) proposed the following estimators using information based on two auxiliary variables $x$ and $z$ for estimating the population mean of the study variable $y$ under different situations given by

$$
\begin{aligned}
& \bar{y}_{\mathrm{SK}}^{1}=\left\{\bar{y}^{*}+b_{y x}^{*}\left(\bar{x}^{\prime}-\bar{x}^{*}\right)\right\} \frac{\bar{Z}}{\left\{\bar{Z}+\eta_{1}\left(\bar{z}^{*}-\bar{Z}\right)\right\}}, \\
& \bar{y}_{\mathrm{SK}}^{2}=\left\{\bar{y}^{*}+b_{y x}^{*}\left(\bar{x}^{\prime}-\bar{x}^{*}\right)\right\} \frac{\bar{z}^{\prime}}{\left\{\bar{z}^{\prime}+\eta_{2}\left(\bar{z}^{*}-\bar{z}^{\prime}\right)\right\}}, \\
& \bar{y}_{\mathrm{SK}}^{3}=\left\{\bar{y}^{*}+b_{y x}^{* *}\left(\bar{x}^{\prime}-\bar{x}\right)\right\} \frac{\bar{Z}}{\left\{\bar{Z}+\eta_{3}(\bar{z}-\bar{Z})\right\}}, \\
& \bar{y}_{\mathrm{SK}}^{4}=\left\{\bar{y}^{*}+b_{y x}^{* *}\left(\bar{x}^{\prime}-\bar{x}\right)\right\} \frac{\bar{z}^{\prime}}{\left\{\bar{z}^{\prime}+\eta_{4}\left(\bar{z}-\bar{z}^{\prime}\right)\right\}},
\end{aligned}
$$

where $\bar{y}^{*}=\left(n_{1} / n\right) \bar{y}_{(1)}+\left(n_{2} / n\right) \bar{y}_{(2)}^{*}, \bar{x}^{*}=\left(n_{1} / n\right) \bar{x}_{(1)}+\left(n_{2} / n\right) \bar{x}_{(2)}^{*}, \bar{z}^{*}=\left(n_{1} / n\right) \bar{z}_{(1)}+\left(n_{2} / n\right) \bar{z}_{(2)}^{*}, \bar{x}^{\prime}=$ $\left(1 / n^{\prime}\right) \sum\left(x_{i}\right), \bar{z}^{\prime}=\left(1 / n^{\prime}\right) \sum\left(z_{i}\right), \bar{x}=(1 / n) \sum\left(x_{i}\right)$, and $\bar{z}=(1 / n) \sum\left(z_{i}\right)$ with $\left(\bar{y}_{(1)}, \bar{x}_{(1)}, \bar{z}_{(1)}\right)$, and $\left(\bar{y}_{(2)}^{*}, \bar{x}_{(2)}^{*}\right.$, $\left.\bar{z}_{(2)}^{*}\right)$ being the sample means based on $n_{1}$ units and sub-sample means based on $r$ units of the variates $(y, x, z)$ respectively and $\eta_{1}, \eta_{2}, \eta_{3}$, and $\eta_{4}$ are suitably chosen constants.

The MSE's of these estimators are given by

$$
\begin{aligned}
\operatorname{MSE}\left(\bar{y}_{\mathrm{SK}}^{1}\right)= & \bar{Y}^{2}\left[f_{n}^{\prime}\left\{C_{y}^{2}+\eta_{1}\left(\eta_{1}-2 K_{y z}\right) C_{z}^{2}\right\}+\left(f_{n}-f_{n}^{\prime}\right)\left\{\left(1-\rho_{y x}^{2}\right) C_{y}^{2}+\eta_{1}\left(\eta_{1}-2 A^{*}\right) C_{z}^{2}\right\}\right. \\
& \left.+g_{n}\left\{C_{y(2)}^{2}+K_{y x}\left(K_{y x}-2 K_{y x(2)}\right) C_{x(2)}^{2}+\eta_{1}\left(\eta_{1}-2 B^{*}\right) C_{z(2)}^{2}\right\}\right],
\end{aligned}
$$




$$
\begin{aligned}
\operatorname{MSE}\left(\bar{y}_{\mathrm{SK}}^{2}\right)= & \bar{Y}^{2}\left[f_{n}^{\prime} C_{y}^{2}+\left(f_{n}-f_{n}^{\prime}\right)\left\{\left(1-\rho_{y x}^{2}\right) C_{y}^{2}+\eta_{2}\left(\eta_{2}-2 A^{*}\right) C_{z}^{2}\right\}\right. \\
& \left.+g_{n}\left\{C_{y(2)}^{2}+K_{y x}\left(K_{y x}-2 K_{y x(2)}\right) C_{x(2)}^{2}+\eta_{2}\left(\eta_{2}-2 B^{*}\right) C_{z(2)}^{2}\right\}\right], \\
\operatorname{MSE}\left(\bar{y}_{\mathrm{SK}}^{3}\right)= & \bar{Y}^{2}\left[f_{n}^{\prime}\left\{C_{y}^{2}+\eta_{3}\left(\eta_{3}-2 K_{y z}\right) C_{z}^{2}\right\}+\left(f_{n}-f_{n}^{\prime}\right)\left\{\left(1-\rho_{y x}^{2}\right) C_{y}^{2}+\eta_{3}\left(\eta_{3}-2 A^{*}\right) C_{z}^{2}\right\}+g_{n} C_{y(2)}^{2}\right], \\
\operatorname{MSE}\left(\bar{y}_{\mathrm{SK}}^{4}\right)= & \bar{Y}^{2}\left[f_{n}^{\prime} C_{y}^{2}+\left(f_{n}-f_{n}^{\prime}\right)\left\{\left(1-\rho_{y x}^{2}\right) C_{y}^{2}+\eta_{4}\left(\eta_{4}-2 A^{*}\right) C_{z}^{2}\right\}+g_{n} C_{y(2)}^{2}\right],
\end{aligned}
$$

where

$$
\begin{aligned}
& C_{x}^{2}=\frac{S_{x}^{2}}{\bar{X}^{2}} \\
& \rho_{y x}=\frac{S_{y x}}{S_{y} S_{x}}, \quad \rho_{y x(2)}=\frac{S_{y x(2)}}{S_{y(2)} S_{x(2)}}, \\
& C_{y}^{2}=\frac{S_{y}^{2}}{\bar{Y}^{2}}, \quad C_{y(2)}^{2}=\frac{S_{y(2)}^{2}}{\bar{Y}_{S}^{2}}, \\
& \rho_{y z}=\frac{S_{y z}}{S_{y} S_{z}}, \\
& K_{y z}=\frac{\rho_{y z} C_{y}}{C_{z}} \\
& \rho_{y z(2)}=\frac{S_{y z(2)}}{S_{y(2)} S_{z(2)}}, \\
& \rho_{x z}=\frac{S_{x z}}{S_{x} S_{z}}, \quad \rho_{x z(2)}=\frac{S_{x z(2)}}{S_{x(2)} S_{z(2)}}, \\
& A^{*}=K_{y z}-K_{y x} K_{x z}, \\
& K_{y z(2)}=\frac{\rho_{y z(2)} C_{y(2)}}{C_{z(2)}}, \\
& K_{y x}=\frac{\rho_{y x} C_{y}}{C_{x}}, \quad K_{y x(2)}=\frac{\rho_{y x(2)} C_{y(2)}}{C_{x(2)}}, \\
& K_{x z}=\frac{\rho_{x z} C_{x}}{C_{z}}, \quad K_{x z(2)}=\frac{\rho_{x z(2)} C_{x(2)}}{C_{z(2)}},
\end{aligned}
$$

The minimum MSE's of the above stated estimators are also given by

$$
\begin{aligned}
& \min \operatorname{MSE}\left(\bar{y}_{\mathrm{SK}}^{1}\right)=\operatorname{MSE}\left(\bar{y}_{\operatorname{Reg}_{(1)}}\right)-\bar{Y}^{2}\left[\frac{B_{1}^{2}}{A_{1}}\right], \\
& \min \operatorname{MSE}\left(\bar{y}_{\mathrm{SK}}^{2}\right)=\operatorname{MSE}\left(\bar{y}_{\operatorname{Reg}_{(1)}}\right)-\bar{Y}^{2}\left[\frac{B_{2}^{2}}{A_{1}}\right], \\
& \min \operatorname{MSE}\left(\bar{y}_{\mathrm{SK}}^{3}\right)=\operatorname{MSE}\left(\bar{y}_{\operatorname{Reg}_{(2)}}\right)-\bar{Y}^{2}\left[\frac{B_{3}^{2}}{A_{2}}\right], \\
& \min \operatorname{MSE}\left(\bar{y}_{\mathrm{SK}}^{4}\right)=\operatorname{MSE}\left(\bar{y}_{\operatorname{Reg}_{(2)}}\right)-\bar{Y}^{2} f^{\prime} A^{* 2} C_{z}^{2},
\end{aligned}
$$

where $B_{1}=f_{n}^{\prime} K_{y z} C_{z}^{2}+\left(f_{n}-f_{n}^{\prime}\right) A^{*} C_{z}^{2}+g_{n} B^{*} C_{z(2)}^{2}, A_{1}=f_{n} C_{z}^{2}+g_{n} C_{z(2)}^{2}, B_{2}=\left(f_{n}-f_{n}^{\prime}\right) A^{*} C_{z}^{2}+g_{n} B^{*} C_{z(2)}^{2}$, $B_{3}=f_{n}^{\prime} K_{y z}+\left(f_{n}-f_{n}^{\prime}\right) A^{*}$, and $A_{2}=f_{n}$.

Further, Shabbir and Khan (2013) proposed the following estimators using information based on two auxiliary variables $x$ and $z$ to estimate the population mean of the study variable $y$ under the different situations given by

$$
\begin{aligned}
& \bar{y}_{\mathrm{SK}}^{5}=\bar{y}^{*}\left(\frac{\bar{x}^{\prime}}{\bar{x}^{*}}\right)^{\alpha_{1}}\left(\frac{\bar{Z}}{\bar{z}^{*}}\right)^{\alpha_{2}}+b_{y x}^{*}\left(\bar{x}^{\prime}-\bar{x}^{*}\right)+b_{y z}^{*}\left(\bar{Z}-\bar{z}^{*}\right), \\
& \bar{y}_{\mathrm{SK}}^{6}=\bar{y}^{*}\left(\frac{\bar{x}^{\prime}}{\bar{x}^{*}}\right)^{\alpha_{3}}\left(\frac{\bar{z}}{\bar{z}^{*}}\right)^{\alpha_{4}}+b_{y x}^{*}\left(\bar{x}^{\prime}-\bar{x}^{*}\right)+b_{y z}^{*}\left(\bar{z}^{\prime}-\bar{z}^{*}\right), \\
& \bar{y}_{\mathrm{SK}}^{7}=\bar{y}^{*}\left(\frac{\bar{x}^{\prime}}{\bar{x}}\right)^{\alpha_{5}}\left(\frac{\bar{Z}}{\bar{z}}\right)^{\alpha_{6}}+b_{y x}^{* *}\left(\bar{x}^{\prime}-\bar{x}\right)+b_{y z}^{* *}(\bar{Z}-\bar{z}), \\
& \bar{y}_{\mathrm{SK}}^{8}=\bar{y}^{*}\left(\frac{\bar{x}^{\prime}}{\bar{x}}\right)^{\alpha_{7}}\left(\frac{\bar{z}^{\prime}}{\bar{z}}\right)^{\alpha_{8}}+b_{y x}^{* *}\left(\bar{x}^{\prime}-\bar{x}\right)+b_{y z}^{* *}\left(\bar{z}^{\prime}-\bar{z}\right),
\end{aligned}
$$

where $\alpha_{1}, \alpha_{2}, \alpha_{3}, \alpha_{4}, \alpha_{5}, \alpha_{6}, \alpha_{7}$, and $\alpha_{8}$ are the characterizing scalars to be chosen suitably and $b_{y x}^{*}=s_{y x}^{*} / s_{x}^{* 2}, b_{y z}^{*}=s_{y z}^{*} / s_{z}^{* 2}, b_{y x}^{* *}=s_{y x}^{*} / s_{x}^{2}, b_{y z}^{* *}=s_{y z}^{* *} / s_{z}^{2}$ are simple regression coefficients. 
The MSE's of these estimators are given by

$$
\begin{aligned}
\operatorname{MSE}\left(\bar{y}_{\mathrm{SK}}^{5}\right)= & \bar{Y}^{2}\left[f_{n} C_{y}^{2}+g_{n} C_{y(2)}^{2}+\left(\alpha_{1}+K_{y x}\right)^{2} m_{1}+\left(\alpha_{2}+K_{y z}\right)^{2} m_{2}-2\left(\alpha_{1}+K_{y x}\right) m_{3}\right. \\
& \left.-2\left(\alpha_{2}+K_{y z}\right) m_{4}+2\left(\alpha_{1}+K_{y x}\right)\left(\alpha_{2}+K_{y z}\right) m_{5}\right], \\
\operatorname{MSE}\left(\bar{y}_{\mathrm{SK}}^{6}\right)= & \bar{Y}^{2}\left[f_{n} C_{y}^{2}+g_{n} C_{y(2)}^{2}+\left(\alpha_{3}+K_{y x}\right)^{2} m_{1}+\left(\alpha_{4}+K_{y z}\right)^{2} m_{2}^{\prime}-2\left(\alpha_{3}+K_{y x}\right) m_{3}\right. \\
& \left.-2\left(\alpha_{4}+K_{y z}\right) m_{4}^{\prime}+2\left(\alpha_{3}+K_{y x}\right)\left(\alpha_{4}+K_{y z}\right) m_{5}\right], \\
\operatorname{MSE}\left(\bar{y}_{\mathrm{SK}}^{7}\right)= & \bar{Y}^{2}\left[f_{n}^{\prime} C_{y}^{2}+g_{n} C_{y(2)}^{2}+\left\{\left(f_{n}-f_{n}^{\prime}\right) C_{y}^{2}+\left(\alpha_{5}+K_{y x}\right)^{2} C_{x}^{2}-2\left(\alpha_{5}+K_{y x}\right) \rho_{y x} C_{y} C_{x}\right.\right. \\
& \left.+2\left(\alpha_{5}+K_{y x}\right)\left(\alpha_{6}+K_{y z}\right) \rho_{x z} C_{x} C_{z}+f_{n}\left\{\left(\alpha_{6}+K_{y z}\right)^{2} C_{z}^{2}-2\left(\alpha_{6}+K_{y z}\right) \rho_{y z} C_{y} C_{z}\right\}\right], \\
\operatorname{MSE}\left(\bar{y}_{\mathrm{SK}}^{8}\right)= & \bar{Y}^{2}\left[f_{n}^{\prime} C_{y}^{2}+g_{n} C_{y(2)}^{2}+\left(f_{n}-f_{n}^{\prime}\right)\left\{C_{y}^{2}+\left(\alpha_{7}+K_{y x}\right)^{2} C_{x}^{2}-2\left(\alpha_{7}+K_{y x}\right) \rho_{y x} C_{y} C_{x}\right.\right. \\
& +\left(\alpha_{8}+K_{y z}\right)^{2} C_{z}^{2}-2\left(\alpha_{8}+K_{y z}\right) \rho_{y z} C_{y} C_{z}+2\left(\alpha_{7}+K_{y x}\right)\left(\alpha_{8}+K_{y z}\right) \rho_{x z} C_{x} C_{z} \\
& \left.+f_{n}\left\{\left(\alpha_{6}+K_{y z}\right)^{2} C_{z}^{2}-2\left(\alpha_{6}+K_{y z}\right) \rho_{y z} C_{y} C_{z}\right\}\right],
\end{aligned}
$$

where

$$
\begin{array}{ll}
m_{1}=\left(f_{n}-f_{n}^{\prime}\right) C_{x}^{2}+g_{n} C_{x(2)}^{2}, & m_{2}=f_{n} C_{z}^{2}+g_{n} C_{z(2)}^{2}, \\
m_{3}=\left(f_{n}-f_{n}^{\prime}\right) \rho_{y x} C_{y} C_{x}+g_{n} \rho_{y x(2)} C_{y(2)} C_{x(2)}, & m_{4}=f_{n} \rho_{y z} C_{y} C_{z}+g_{n} \rho_{y z(2)} C_{y(2)} C_{z(2)}, \\
m_{5}=\left(f_{n}-f_{n}^{\prime}\right) \rho_{x z} C_{x} C_{z}+g_{n} \rho_{x z(2)} C_{x(2)} C_{z(2)}, & m_{2}^{\prime}=\left(f_{n}-f_{n}^{\prime}\right) C_{z}^{2}+g_{n} C_{z(2)}^{2}, \\
m_{4}^{\prime}=\left(f_{n}-f_{n}^{\prime}\right) \rho_{y z} C_{y} C_{z}+g_{n} \rho_{y z(2)} C_{y(2)} C_{z(2)} . &
\end{array}
$$

Also, the minimum MSE's of the above stated estimators are given by

$$
\begin{aligned}
& \min \operatorname{MSE}\left(\bar{y}_{\mathrm{SK}}^{5}\right)=\operatorname{MSE}\left(\bar{y}^{*}\right)-\bar{Y}^{2}\left\{\frac{m_{2} m_{3}^{2}+m_{1} m_{4}^{2}-2 m_{3} m_{4} m_{5}}{m_{1} m_{2}-m_{5}^{2}}\right\}, \\
& \min \operatorname{MSE}\left(\bar{y}_{\mathrm{SK}}^{6}\right)=\operatorname{MSE}\left(\bar{y}^{*}\right)-\bar{Y}^{2}\left\{\frac{m_{2}^{\prime} m_{3}^{2}+m_{1} m_{4}^{\prime 2}-2 m_{3} m_{4} m_{5}}{m_{1} m_{2}^{\prime}-m_{5}^{2}}\right\}, \\
& \min \operatorname{MSE}\left(\bar{y}_{\mathrm{SK}}^{7}\right)=\operatorname{MSE}\left(\bar{y}^{*}\right)-\bar{Y}^{2}\left[\frac{\left\{f_{n} \rho_{y z}^{2}+f_{n}^{\prime}\left(\rho_{y x}^{2}-2 \rho_{y x} \rho_{y z} \rho_{x z}\right)\right\}}{\left\{f_{n}-\left(f_{n}-f_{n}^{\prime}\right) \rho_{y x}^{2}\right\}}\right], \\
& \min \operatorname{MSE}\left(\bar{y}_{\mathrm{SK}}^{8}\right)=\operatorname{MSE}\left(\bar{y}^{*}\right)-\bar{Y}^{2} f_{n}^{\prime} C_{y}^{2}\left\{\frac{\rho_{y x}^{2}+\rho_{y z}^{2}-2 \rho_{y x} \rho_{y z} \rho_{x z}}{\left(1-\rho_{x z}^{2}\right)}\right\} .
\end{aligned}
$$

Bhushan and Naqvi (2015) similarly proposed some generalized classes of estimators in presence of non-response using two auxiliary information given by

$$
\begin{array}{ll}
t_{(\mathrm{BN})}^{1}=f_{1}\left(\bar{y}^{*}, \bar{x}^{*}, \bar{x}^{\prime}, u\right), & t_{(\mathrm{BN})}^{2}=f_{2}\left(\bar{y}^{*}, \bar{x}^{*}, \bar{x}^{\prime}, \bar{z}^{*}, \bar{z}^{\prime}\right), \\
t_{(\mathrm{BN})}^{3}=f_{3}\left(\bar{y}^{*}, \bar{x}^{*}, \bar{x}^{\prime}, v\right), & t_{(\mathrm{BN})}^{4}=f_{4}\left(\bar{y}^{*}, \bar{x}, \bar{x}^{\prime}, \bar{z}, \bar{z}^{\prime}\right),
\end{array}
$$


where $u=\bar{z}^{*} / \bar{Z}$ and $v=\bar{z} / \bar{Z}$ and these estimators satisfying the following conditions.

$$
\begin{aligned}
& f_{1}(\bar{Y}, \bar{X}, \bar{X}, 1)=\bar{Y}, \quad f_{1}^{0}=1, \quad f_{1}^{1}=-f_{1}^{2}, \quad f_{2}(\bar{Y}, \bar{X}, \bar{X}, \bar{Z}, \bar{Z})=\bar{Y}, \quad f_{2}^{0}=1, \quad f_{2}^{1}=-f_{2}^{2}, \\
& f_{2}^{3}=-f_{2}^{4}, \quad f_{3}(\bar{Y}, \bar{X}, \bar{X}, 1)=\bar{Y}, \quad f_{3}^{0}=1, \quad f_{3}^{1}=-f_{3}^{2}, \quad f_{4}(\bar{Y}, \bar{X}, \bar{X}, \bar{Z}, \bar{Z})=\bar{Y}, \quad f_{4}^{0}=1, \\
& f_{4}^{1}=-f_{4}^{2}, \quad \text { and } f_{4}^{3}=-f_{4}^{4} .
\end{aligned}
$$

The MSE's and minimum MSE's are given by

$$
\begin{aligned}
& \operatorname{MSE}\left(t_{(\mathrm{BN})}^{1}\right)=\operatorname{MSE}\left(\bar{y}^{*}\right)+\eta_{6}\left(f_{1}^{3}\right)^{2}+2 \bar{Y} \eta_{7} f_{1}^{3}+\bar{X}^{2} \eta_{3}\left(f_{1}^{1}\right)^{2}+2 \bar{Y} \bar{X} \eta_{8} f_{1}^{1}+2 \bar{X} \eta_{9} f_{1}^{1} f_{1}^{3}, \\
& \operatorname{MSE}\left(t_{(\mathrm{BN})}^{2}\right)=\operatorname{MSE}\left(\bar{y}^{*}\right)+\bar{X}^{2} \eta_{3}\left(f_{2}^{1}\right)^{2}+\bar{Z}^{2} \eta_{10}\left(f_{2}^{3}\right)^{2}+2 \bar{X} \bar{Z} f_{2}^{1} f_{2}^{3}+2 \bar{Y} \bar{X} \eta_{8} f_{2}^{1}+2 \bar{Y} \bar{Z} \eta_{11} f_{2}^{3}, \\
& \operatorname{MSE}\left(t_{(\mathrm{BN})}^{3}\right)=\operatorname{MSE}\left(\bar{y}^{*}\right)+\bar{X}^{2} \eta_{3}^{\prime}\left(f_{3}^{1}\right)^{2}+\eta_{4}\left(f_{3}^{3}\right)^{2}+2 \bar{X} \eta_{9}^{\prime} f_{3}^{1} f_{3}^{3}+2 \bar{Y} \bar{X} \eta_{8}^{\prime} f_{3}^{1}+2 \bar{Y} \eta_{5} f_{3}^{3}, \\
& \operatorname{MSE}\left(t_{(\mathrm{BN})}^{4}\right)=\operatorname{MSE}\left(\bar{y}^{*}\right)+\bar{X}^{2} \eta_{3}^{\prime}\left(f_{4}^{1}\right)^{2}+\bar{Z}^{2} \eta_{10}\left(f_{4}^{3}\right)^{2}+2 \bar{X} \bar{Z} \eta_{9}^{\prime} f_{4}^{1} f_{4}^{3}+2 \bar{Y} \bar{X} \eta_{8}^{\prime} f_{4}^{1}+2 \bar{Y} \bar{Z} \eta_{11}^{\prime} f_{4}^{3},
\end{aligned}
$$

where

$$
\begin{array}{ll}
m_{6}=f_{n} C_{z}^{2}, & m_{7}=f_{n} \rho_{y z} C_{y} C_{z}, \\
m_{1}^{\prime}=\left(f_{n}-f_{n}^{\prime}\right) C_{x}^{2}, & m_{3}^{\prime}=\left(f_{n}-f_{n}^{\prime}\right) \rho_{y x} C_{y} C_{x}, \\
m_{5}^{\prime}=\left(f_{n}-f_{n}^{\prime}\right) \rho_{x z} C_{x} C_{z}, & m_{6}^{\prime}=\left(f_{n}-f_{n}^{\prime}\right) C_{z}^{2}, \\
m_{7}^{\prime}=\left(f_{n}-f_{n}^{\prime}\right) \rho_{y z} C_{y} C_{z} . &
\end{array}
$$

The minimum MSE's of the above stated estimators are given by

$$
\begin{aligned}
& \min \operatorname{MSE}\left(t_{(\mathrm{BN})}^{1}\right)=\operatorname{MSE}\left(\bar{y}^{*}\right)-\bar{Y}^{2}\left\{\frac{\left(2 m_{3} m_{4} m_{5}-m_{2} m_{3}^{2}-m_{1} m_{4}^{2}\right)}{\left(m_{5}^{2}-m_{1} m_{2}\right)}\right\}, \\
& \min \operatorname{MSE}\left(t_{(\mathrm{BN})}^{2}\right)=\operatorname{MSE}\left(\bar{y}^{*}\right)-\bar{Y}^{2}\left\{\frac{\left(2 m_{3} m_{4}^{\prime} m_{5}-m_{2}^{\prime} m_{3}^{2}-m_{1} m_{4}^{\prime 2}\right)}{\left(m_{5}^{2}-m_{1} m_{2}^{\prime}\right)}\right\}, \\
& \min \operatorname{MSE}\left(t_{(\mathrm{BN})}^{3}\right)=\operatorname{MSE}\left(\bar{y}^{*}\right)-\bar{Y}^{2}\left\{\frac{\left(2 m_{7} m_{3}^{\prime} m_{5}^{\prime}-m_{1}^{\prime} m_{7}^{2}-m_{6} m_{3}^{\prime 2}\right)}{\left(m_{5}^{\prime 2}-m_{1}^{\prime} m_{6}\right)}\right\}, \\
& \min \operatorname{MSE}\left(t_{(\mathrm{BN})}^{4}\right)=\operatorname{MSE}\left(\bar{y}^{*}\right)-\bar{Y}^{2}\left\{\frac{\left(2 m_{3}^{\prime} m_{5}^{\prime} m_{7}^{\prime}-m_{1}^{\prime} m_{7}^{\prime 2}-m_{6}^{\prime} m_{3}^{\prime 2}\right)}{\left(m_{5}^{\prime 2}-m_{1}^{\prime} m_{6}^{\prime}\right)}\right\} .
\end{aligned}
$$

\section{Proposed estimators}

Let

$$
\begin{aligned}
t & =\bar{y}^{*}, \\
\operatorname{MSE}\left(\bar{y}^{*}\right) & =\bar{Y}^{2}\left\{f_{n} C_{y}^{2}+g_{n} C_{y(2)}^{2}\right\} .
\end{aligned}
$$

Searls (1964) proposed a useful technique to improve the efficiency of any estimator.

Let us consider the Searls type estimators under non-response given by

$$
T=\alpha t
$$


so that

$$
\operatorname{MSE}(T)=\frac{\bar{Y}^{2} \operatorname{MSE}(t)}{\bar{Y}^{2}+\operatorname{MSE}(t)}
$$

It can be easily observed that the minimum MSE of $T$ is always lesser than $t$.

Following Singh and Kumar (2010b), Shabbir and Khan (2013) and Bhushan and Naqvi (2015) by using the Searls type transformation, we propose the following efficient estimators using information based on two auxiliary variables for estimating the population mean of the study variable in presence of a non-response under the strategies given below.

- Strategy I: In this strategy, we assume that non-response occurs on the study variable as well as on the auxiliary variable and the population mean $\bar{X}$ of the first auxiliary variable $x$ is unknown; however, the population mean $\bar{Z}$ of the second auxiliary variable $z$ is known. The proposed estimator for estimating the population mean is

$$
T_{(\mathrm{BP})}^{1}=\gamma_{1} \bar{y}^{*}+\theta_{1}\left(\bar{x}^{*}-\bar{x}^{\prime}\right)+\omega_{1}\left(\bar{z}^{*}-\bar{Z}\right)
$$

- Strategy II: In this strategy, we assume that non-response occurs on the study variable as well as on the auxiliary variable and the population mean $\bar{X}$ of first auxiliary variable $x$ as well as the population mean $\bar{Z}$ of the second auxiliary variable $z$ if both are unknown. The proposed estimator for estimating the population mean is given by

$$
T_{(\mathrm{BP})}^{2}=\gamma_{2} \bar{y}^{*}+\theta_{2}\left(\bar{x}^{*}-\bar{x}^{\prime}\right)+\omega_{2}\left(\bar{z}^{*}-\bar{z}^{\prime}\right) .
$$

- Strategy III: In this strategy, we assume that non-response occurs on the study variable only and the population mean $\bar{X}$ of first auxiliary variable $x$ is unknown; however, the population mean $\bar{Z}$ of the second auxiliary variable $z$ is known. The proposed estimator for estimating the population mean is

$$
T_{(\mathrm{BP})}^{3}=\gamma_{3} \bar{y}^{*}+\theta_{3}\left(\bar{x}-\bar{x}^{\prime}\right)+\omega_{3}(\bar{z}-\bar{Z})
$$

- Strategy IV: In this strategy, we assume that non-response occurs on the study variable only and the population mean if both the auxiliary variables are unknown. The proposed estimator under this strategy is

$$
T_{(\mathrm{BP})}^{4}=\gamma_{4} \bar{y}^{*}+\theta_{4}\left(\bar{x}-\bar{x}^{\prime}\right)+\omega_{4}\left(\bar{z}-\bar{z}^{\prime}\right) .
$$

Theorem 1. The bias and minimum mean square error of the proposed estimators $T_{(B P)}^{k}, k=1,2,3,4$ are given by

$$
\begin{aligned}
\operatorname{Bias}\left(T_{(B P)}^{k}\right) & =\left(\gamma_{k}-1\right) \bar{Y}, \\
\min \operatorname{MSE}\left(T_{(B P)}^{k}\right) & =\frac{\bar{Y}^{2} \min \operatorname{MSE}\left(t_{(B N)}^{k}\right)}{\bar{Y}^{2}+\min \operatorname{MSE}\left(t_{(B N)}^{k}\right)} .
\end{aligned}
$$

Proof: Now, for the bias amd MSE of $T_{(\mathrm{BP})}^{1}$, we have

$$
T_{(\mathrm{BP})}^{1}=\gamma_{1} \bar{Y}\left(1+\varepsilon_{0}^{*}\right)+\theta_{1}\left\{\bar{X}\left(1+\varepsilon_{1}^{*}\right)-\bar{X}\left(1+\varepsilon_{1}^{\prime}\right)\right\}+\omega_{1}\left\{\bar{Z}\left(1+\varepsilon_{2}^{*}\right)-\bar{Z}\right\} .
$$


Thus,

$$
T_{(\mathrm{BP})}^{1}-\bar{Y}=\left(\gamma_{1}-1\right) \bar{Y}+\gamma_{1} \bar{Y} \varepsilon_{0}^{*}+\theta_{1} \bar{X}\left(\varepsilon_{1}^{*}-\varepsilon_{1}^{\prime}\right)+\omega_{1} \bar{Z} \varepsilon_{2}^{*}
$$

for bias taking expectation on both sides, we get

$$
\operatorname{Bias}\left(T_{(\mathrm{BP})}^{1}\right)=\left(\gamma_{1}-1\right) \bar{Y}
$$

for MSE, squaring and taking expectation on both sides of the above equation, we get

$$
\begin{aligned}
\operatorname{MSE}\left(T_{(\mathrm{BP})}^{1}\right)= & \left(\gamma_{1}-1\right)^{2} \bar{Y}^{2}+\gamma_{1}^{2} \bar{Y}^{2}\left\{f_{n} C_{y}^{2}+g_{n} C_{y(2)}^{2}\right\}+\theta_{1}^{2} \bar{X}^{2}\left\{\left(f_{n}-f_{n}^{\prime}\right) C_{x}^{2}+g_{n} C_{x(2)}^{2}\right\} \\
& +\omega_{1}^{2} \bar{Z}^{2}\left\{f_{n} C_{z}^{2}+g_{n} C_{z(2)}^{2}\right\}+2 \gamma_{1} \theta_{1} \bar{X} \bar{Y}\left\{\left(f_{n}-f_{n}^{\prime}\right) \rho_{y x} C_{y} C_{x}+g_{n} \rho_{y x(2)} C_{y(2)} C_{x(2)}\right\} \\
& +2 \gamma_{1} \omega_{1} \bar{Y} \bar{Z}\left\{f_{n} \rho_{y z} C_{y} C_{z}+g_{n} \rho_{y z(2)} C_{y(2)} C_{z(2)}\right\}+2 \theta_{1} \omega_{1} \bar{X} \bar{Z}\left\{\left(f_{n}-f_{n}^{\prime}\right) \rho_{x z} C_{x} C_{z}\right. \\
& \left.+g_{n} \rho_{x z(2)} C_{x(2)} C_{z(2)}\right\} \\
\operatorname{MSE}\left(T_{(\mathrm{BP})}^{1}\right)= & \bar{Y}^{2}+\gamma_{1}^{2} \bar{Y}^{2}+\gamma_{1}^{2} \operatorname{Var}\left(\bar{y}^{*}\right)-2 \gamma_{1} \bar{Y}^{2}+\theta_{1}^{2} \bar{X}^{2} m_{1}+\omega_{1}^{2} \bar{Z}^{2} m_{2}+2 \gamma_{1} \theta_{1} \bar{X} \bar{Y} m_{3} \\
& +2 \gamma_{1} \omega_{1} \bar{Y} \bar{Z} m_{4}+2 \theta_{1} \omega_{1} \bar{X} \bar{Z} m_{5} .
\end{aligned}
$$

Differentiating $\operatorname{MSE}\left(T_{(\mathrm{BP})}^{1}\right)$, for optimum value of the $\gamma_{1}, \theta_{1}$, and $\omega_{1}$, partially with respect to $\gamma_{1}$, $\theta_{1}, \omega_{1}$, and equating to zero. we get the optimum value of $\gamma_{1}, \theta_{1}$, and $\omega_{1}$ are given by

$$
\frac{\partial \operatorname{MSE}\left(T_{(\mathrm{BP})}^{1}\right)}{\partial \gamma_{1}}=0, \quad \frac{\partial \operatorname{MSE}\left(T_{(\mathrm{BP})}^{1}\right)}{\partial \theta_{1}}=0, \quad \text { and } \quad \frac{\partial \operatorname{MSE}\left(T_{(\mathrm{BP})}^{1}\right)}{\partial \omega_{1}}=0
$$

so that

$$
\gamma_{1}=\frac{\bar{Y}^{2}-\theta_{1} \bar{X} \bar{Y} m_{3}-\omega_{1} \bar{Y} \bar{Z} m_{4}}{\bar{Y}^{2}+A}, \quad \theta_{1}=\frac{-\gamma_{1} \bar{Y} m_{3}-\omega_{1} \bar{Z} m_{5}}{\bar{X} m_{1}}, \quad \omega_{1}=\frac{-\gamma_{1} \bar{Y} m_{4}-\theta_{1} \bar{X} m_{5}}{\bar{Z} m_{2}}
$$

solving these equations, we get

$$
\begin{aligned}
\gamma_{\text {lopt }} & =\frac{\bar{Y}^{2}}{\bar{Y}^{2}+A-\bar{Y}^{2}\left\{\frac{m_{2} m_{3}^{2}+m_{1} m_{4}^{2}-2 m_{3} m_{4} m_{5}}{m_{1} m_{2}-m_{5}^{2}}\right\}}, \\
\theta_{\text {lopt }} & =\gamma_{1 \mathrm{opt}}\left(\frac{\bar{Y}}{\bar{X}}\right)\left\{\frac{m_{4} m_{5}-m_{2} m_{3}}{m_{1} m_{2}-m_{5}^{2}}\right\}, \\
\omega_{\text {lopt }} & =\gamma_{\text {lopt }}\left(\frac{\bar{Y}}{\bar{Z}}\right)\left\{\frac{m_{3} m_{5}-m_{1} m_{4}}{m_{1} m_{2}-m_{5}^{2}}\right\} .
\end{aligned}
$$

By using these optimum values of $\gamma_{1}, \theta_{1}$, and $\omega_{1}$ in $\operatorname{MSE}\left(T_{(\mathrm{BP})}^{1}\right)$, we get the minimum mean square error as

$$
\min \operatorname{MSE}\left(T_{(\mathrm{BP})}^{1}\right)=\frac{\bar{Y}^{2}\left[A-\bar{Y}^{2}\left\{\frac{m_{2} m_{3}^{2}+m_{1} m_{4}^{2}-2 m_{3} m_{4} m_{5}}{m_{1} m_{2}-m_{5}^{2}}\right\}\right]}{\bar{Y}^{2}+\left[A-\bar{Y}^{2}\left\{\frac{m_{2} m_{3}^{2}+m_{1} m_{4}^{2}-2 m_{3} m_{4} m_{5}}{m_{1} m_{2}-m_{5}^{2}}\right\}\right]},
$$


where $A=\operatorname{MSE}\left(\bar{y}^{*}\right)$

$$
\min \operatorname{MSE}\left(T_{(\mathrm{BP})}^{1}\right)=\frac{\bar{Y}^{2} \min \cdot \operatorname{MSE}\left(t_{(\mathrm{BN})}^{1}\right)}{\bar{Y}^{2}+\min \operatorname{MSE}\left(t_{(\mathrm{BN})}^{1}\right)}
$$

The derivation of $\operatorname{MSE}\left(T_{(\mathrm{BP})}^{i}\right), i=2,3,4$ can easily be done on similar lines so that the optimum values of characterizing scalars are

$$
\begin{aligned}
& \gamma_{2 \mathrm{opt}}=\frac{\bar{Y}^{2}}{\bar{Y}^{2}+A-\bar{Y}^{2}\left\{\frac{m_{2}^{\prime} m_{3}^{2}+m_{1} m_{4}^{\prime 2}-2 m_{3} m_{4}^{\prime} m_{5}}{m_{1} m_{2}^{\prime}-m_{5}^{2}}\right\}}, \\
& \theta_{2 \mathrm{opt}}=\gamma_{2 \mathrm{opt}}\left(\frac{\bar{Y}}{\bar{X}}\right)\left\{\frac{m_{4}^{\prime} m_{5}-m_{2}^{\prime} m_{3}}{m_{1} m_{2}^{\prime}-m_{5}^{2}}\right\}, \\
& \omega_{2 \mathrm{opt}}=\gamma_{2 \mathrm{opt}}\left(\frac{\bar{Y}}{\bar{Z}}\right)\left\{\frac{m_{3} m_{5}-m_{1} m_{4}^{\prime}}{m_{1} m_{2}^{\prime}-m_{5}^{2}}\right\}, \\
& \gamma_{3 \mathrm{opt}}=\frac{\bar{Y}^{2}}{\bar{Y} 2+A-\bar{Y}^{2}\left\{\frac{m_{1}^{\prime} m_{7}^{2}+m_{6} m_{3}^{\prime 2}-2 m_{7} m_{3}^{\prime} m_{5}^{\prime}}{m_{1}^{\prime} m_{6}-m_{5}^{\prime 2}}\right\}} \\
& \theta_{3 \mathrm{opt}}=\gamma_{3 \mathrm{opt}}\left(\frac{\bar{Y}}{\bar{X}}\right)\left\{\frac{m_{5}^{\prime} m_{7}-m_{3}^{\prime} m_{6}}{m_{1}^{\prime} m_{6}-m_{5}^{\prime 2}}\right\}, \\
& \omega_{3 \mathrm{opt}}=\gamma_{3 \mathrm{opt}}\left(\frac{\bar{Y}}{\bar{Z}}\right)\left\{\frac{m_{3}^{\prime} m_{5}-m_{1}^{\prime} m_{7}}{m_{1}^{\prime} m_{6}-m_{5}^{\prime 2}}\right\}, \\
& \gamma_{4 \mathrm{opt}}=\frac{\bar{Y}^{2}}{\bar{Y} 2+A--\bar{Y}^{2}\left\{\frac{m_{1}^{\prime} m_{7}^{\prime 2}+m_{6}^{\prime} m_{3}^{\prime 2}-2 m_{3}^{\prime} m_{5}^{\prime} m_{7}^{\prime}}{m_{1}^{\prime} m_{6}^{\prime}-m_{5}^{\prime 2}}\right\}}, \\
& \theta_{4 \mathrm{opt}}= \gamma_{4 \mathrm{opt}}\left(\frac{\bar{Y}}{\bar{X}}\right)\left\{\frac{m_{5}^{\prime} m_{7}^{\prime}-m_{3}^{\prime} m_{6}^{\prime}}{m_{1}^{\prime} m_{6}^{\prime}-m_{5}^{\prime 2}}\right\}, \\
& \omega_{4 \mathrm{opt}}=\gamma_{4 \mathrm{opt}}\left(\frac{\bar{Y}}{\bar{Z}}\right)\left\{\frac{m_{3}^{\prime} m_{5}^{\prime}-m_{1}^{\prime} m_{7}^{\prime}}{m_{1}^{\prime} m_{6}^{\prime}-m_{5}^{\prime 2}}\right\} .
\end{aligned}
$$

Corollary 1. The proposed estimators $T_{(B P)}^{k}, k=1,2,3,4$ has always lesser MSE than the conventional estimators $t_{(B N)}^{k}, k=1,2,3,4$. In other words,

$$
\min \operatorname{MSE}\left(T_{(B P)}^{k}\right) \leq \min \operatorname{MSE}\left(t_{(B N)}^{k}\right), \quad i=1,2,3,4 .
$$

Proof: Since $\bar{Y}^{2} /\left\{\bar{Y}^{2}+\operatorname{MSE}\left(t_{B N}^{k}\right)\right\}<1, i=1,2,3,4$. Hence the prove.

\section{An empirical study}

We have conducted an empirical study on 2 population described below along with their key parameters. 
1. Data considered from the book "Advanced sampling theory with applications" by Sarjinder Singh (p.1114). The first 30\% (i.e., 21) units have been considered as non-response units.

Fish caught: Estimated number of fish caught by marine boats. Descriptive parameters of the population:

$$
\begin{aligned}
& Y=\text { Estimated number of fish caught during 1995, } \\
& X=\text { Estimated number of fish caught during 1993, } \\
& Z=\text { Estimated number of fish caught during 1992, } \\
& N=69, \quad n^{\prime}=55, \quad n=20, \quad \bar{Y}=4514.899, \quad \bar{Z}=4230.174, \quad \bar{X}=4591.072, \\
& C_{y}=1.3509, \quad C_{z}=1.3164, \quad C_{x}=1.3755, \quad C_{y(2)}=1.3850, \quad C_{z(2)}=1.3640, \\
& C_{x(2)}=1.4160, \quad \rho_{y x}=0.9564, \quad \rho_{y z}=0.9538, \quad \rho_{x z}=0.9632, \quad \rho_{y x(2)}=0.9674, \\
& \rho_{y z(2)}=0.9668, \quad \rho_{x z(2)}=0.9699 .
\end{aligned}
$$

2. The present data belong to the data on physical growth of upper socio-economic group of 95 school going children of Varanasi under an ICMR study, Department of Pediatrics, BHU during 19831984 has been taken under study, (Khare and Sinha, 2007). The first 25\% (i.e., 24 children) units have been considered as non-response units. The value of parameters related to the study character $y$ (the weight of children in $\mathrm{kg}$ ) and the auxiliary character $x$ (skull circumference of the children in $\mathrm{cm}$ ) and additional auxiliary character $z$ (chest circumference of the children in $\mathrm{cm}$ ) have been given as:

$$
\begin{aligned}
& N=95, \quad n^{\prime}=70, \quad n=35, \quad \bar{Y}=19.4968, \quad \bar{Z}=55.8611, \quad \bar{X}=51.1726, \quad C_{y}=0.15613, \\
& C_{z}=0.05860, \quad C_{x}=0.03006, \quad C_{y(2)}=0.12075, \quad C_{z(2)}=0.05402, \quad C_{x(2)}=0.02478, \\
& \rho_{y x}=0.328, \quad \rho_{y z}=0.846, \quad \rho_{x z}=0.297, \quad \rho_{y x(2)}=0.477, \quad \rho_{y z(2)}=0.729, \quad \rho_{x z(2)}=0.570 .
\end{aligned}
$$

The percentage relative efficiency (PRE) of the proposed estimator is calculated by

$$
\operatorname{PRE}=\frac{\operatorname{Var}\left(\bar{y}^{*}\right)}{\min \mathrm{MSE}(\text { conventional and proposed estimators })} \times 100 .
$$

Tables 1 and 2 suggests that the proposed estimator $T_{(\mathrm{BP})}^{1}$ under strategy 1 is more efficient than the Hansen-Hurwitz estimator $\bar{y}^{*}$, conventional ratio and regression estimators $\bar{y}_{R(1)}$ and $\bar{y}_{\operatorname{Reg}(1)}$, Singh and Kumar estimator $\bar{y}_{\mathrm{SK}}^{1}$, Shabbir and Khan estimator $\bar{y}_{\mathrm{SK}}^{5}$ and Bhushan and Naqvi estimator $t_{(\mathrm{BN})}^{1}$ for the different values of $k=2,3,4$. Under the Strategy 2, 3, and 4 the proposed estimators $T_{(\mathrm{BP})}^{2}, T_{(\mathrm{BP})}^{3}$, and $T_{(\mathrm{BP})}^{4}$ are also more efficient than the all conventional estimators along with respective strategy.

\section{Conclusion}

This paper shows that the proposed estimators using the Searls (1964) philosophy in presence of bivariate auxiliary information perform better than the estimators suggested by Singh and Kumar (2010b), Shabbir and Khan (2013) and Bhushan and Naqvi (2015) in terms of PRE. This fact has been also supported through an empirical study. Further, the proposed estimators are important because they provide an improvement over the regression estimators, which are BLUEs. The result of this paper is quite illuminating, both theoretically and empirically. 
Table 1: MSE and PRE of the estimators with respect to $\bar{y}^{*}$ for population 1

\begin{tabular}{|c|c|c|c|c|}
\hline & \multirow{2}{*}{ Estimator } & \multicolumn{3}{|c|}{$1 / k$} \\
\hline & & $1 / 2$ & $1 / 3$ & $1 / 4$ \\
\hline & $\bar{y}^{*}$ & $1915872(100)$ & $2510898(100)$ & $3105923(100)$ \\
\hline & $\bar{y}_{R(1)}$ & $282681.3(677.749)$ & $322643.4(778.227)$ & $362605.6(856.557)$ \\
\hline & $\bar{y}_{R(2)}$ & $837745.0(228.694)$ & $1432771(175.248)$ & 2027797(153.167) \\
\hline & $\bar{y}_{\operatorname{Reg}_{(1)}}$ & 276385.4(693.188) & $314578.8(798.177)$ & $352772.3(880.433)$ \\
\hline & $\bar{y}_{\operatorname{Reg}_{(2)}}$ & $833217.8(229.936)$ & $1428243.7(175.803)$ & $2023269.5(153.510)$ \\
\hline \multirow{4}{*}{ Strategy I } & $\bar{y}_{\mathrm{SK}}^{1}$ & $296847.3(845.855)$ & $335450.7(925.896)$ & $0.1239(231.661)$ \\
\hline & $\bar{y}_{S K}^{5}$ & $141773.2(1351.363)$ & $173677.5(1445.724)$ & $205166.8(1513.853)$ \\
\hline & $t_{(\mathrm{BN})}^{1}$ & 141773.2(1351.363) & $173677.5(1445.724)$ & $205166.8(1513.853)$ \\
\hline & $T_{(\mathrm{BP})}^{1}$ & $140794.0(\mathbf{1 3 6 0 . 7 6 2})$ & $172210.2(\mathbf{1 4 5 8 . 0 4 2})$ & 203122.4(1529.09) \\
\hline \multirow{4}{*}{ Strategy II } & $\bar{y}_{\mathrm{SK}}^{2}$ & $274526.2(697.882)$ & $311980.6(804.825)$ & $349432.1(888.849)$ \\
\hline & $\bar{y}_{\text {SK }}^{6}$ & $250802.88(763.895)$ & $280812.98(894.153)$ & $310819.87(999.268)$ \\
\hline & $t_{(\mathrm{BN})}^{2}$ & $250802.9(763.895)$ & 280813(894.1529) & 310819.9(999.268) \\
\hline & $T_{(\mathrm{BP})}^{2}$ & 247754.6(773.294) & 276997.1(906.470) & $306151.7(\mathbf{1 0 1 4 . 5 0 5})$ \\
\hline \multirow{4}{*}{ Strategy III } & $\bar{y}_{\mathrm{SK}}^{3}$ & $811473.8(236.098)$ & $1406500(178.521)$ & $2001526(155.178)$ \\
\hline & $\bar{y}_{\mathrm{SK}}^{y^{\prime}}$ & $704277.1(272.0338)$ & 1299303(193.2496) & $1894329(163.959)$ \\
\hline & $t_{(\mathrm{BN})}^{3}$ & $704277.1(272.0338)$ & 1299303(193.2496) & $1894329(163.959)$ \\
\hline & $T_{(\mathrm{BP})}^{3}$ & 680757.0(281.4325) & 1221448(205.5674) & 173256(179.196) \\
\hline \multirow{4}{*}{ Strategy IV } & $\bar{y}_{\mathrm{SK}}^{4}$ & $831960.3(230.284)$ & $1426986(175.958)$ & 2022012(153.606) \\
\hline & $\bar{y}_{S K}^{8}$ & $815811.2(234.843)$ & $1410837(177.972)$ & 2005863(154.842) \\
\hline & $t_{(\mathrm{BN})}^{4}$ & $815811.2(234.843)$ & $1410837(177.972)$ & $2005863(154.842)$ \\
\hline & $T_{(\mathrm{BP})}^{4}$ & 784417.6(244.241) & 1319511(190.29) & 1826164(170.079) \\
\hline
\end{tabular}

$\mathrm{MSE}=$ mean squared error; $\mathrm{PRE}=$ percentage relative efficiency.

Table 2: MSE and PRE of the estimators with respect to $\bar{y}^{*}$ for population 2

\begin{tabular}{|c|c|c|c|c|}
\hline & \multirow{2}{*}{ Estimator } & \multicolumn{3}{|c|}{$1 / k$} \\
\hline & & $1 / 2$ & $1 / 3$ & $1 / 4$ \\
\hline & $\bar{y}^{*}$ & $0.207214(100)$ & $0.24722(100)$ & $0.287225(100)$ \\
\hline & $\bar{y}_{R(1)}$ & $0.1893(109.489)$ & $0.2231(110.805)$ & $0.2569(104.289)$ \\
\hline & $\bar{y}_{R(2)}$ & $0.1954(106.045)$ & $0.2354(105.018)$ & $0.2754(104.289)$ \\
\hline & $\bar{y}_{\operatorname{Reg}_{(1)}}$ & $0.1845(112.210)$ & $0.2161(114.415)$ & $0.2476(115.992)$ \\
\hline & $\bar{y}_{\operatorname{Reg}_{(2)}}$ & $0.1930(107.380)$ & $0.2330(106.113)$ & $0.2730(105.217)$ \\
\hline \multirow{4}{*}{ Strategy I } & $\bar{y}_{S K}^{1}$ & $0.0789(262.596)$ & $0.1019(242.622)$ & $0.1239(231.661)$ \\
\hline & $\bar{y}_{S K}^{S^{n}}$ & $0.0679(304.874)$ & $0.0880(280.615)$ & $0.1076(266.708)$ \\
\hline & $t_{(\mathrm{BN})}^{1}$ & $0.0679(304.874)$ & $0.0881(280.615)$ & $0.1018(266.708)$ \\
\hline & $T_{(\mathrm{BP})}^{1}$ & $0.0679(\mathbf{3 0 4 . 9 2 9})$ & $0.0888(\mathbf{2 8 0 . 6 8 1})$ & $0.1077(\mathbf{2 6 6 . 7 8 4})$ \\
\hline \multirow{4}{*}{ Strategy II } & $\bar{y}_{\mathrm{SK}}^{2}$ & $0.1155(179.405)$ & $0.1364(181.270)$ & $0.1571(182.857)$ \\
\hline & $\bar{y}_{\mathrm{SK}}^{6 \mathrm{~K}}$ & $0.1014(204.173)$ & $0.1230(200.841)$ & $0.1437(199.851)$ \\
\hline & $t_{(\mathrm{BN})}^{2}$ & $0.0926(223.688)$ & $0.1124(219.768)$ & $0.1318(217.844)$ \\
\hline & $T_{(\mathrm{BP})}^{2}$ & $0.0926(\mathbf{2 2 3 . 7 4 2})$ & $0.1125(\mathbf{2 1 9 . 8 3 2})$ & $0.1318(\mathbf{2 1 7 . 9 1 9})$ \\
\hline \multirow{4}{*}{ Strategy III } & $\bar{y}_{\text {SK }}^{3}$ & $0.0941(220.152)$ & $0.1341(184.315)$ & $0.1741(164.944)$ \\
\hline & $\bar{y}_{S K}^{P^{K}}$ & $0.0867(238.995)$ & $0.1267(195.110)$ & $0.1667(172.286)$ \\
\hline & $t_{(\mathrm{BN})}^{3 \mathrm{~K}}$ & $0.0519(399.098)$ & $0.0919(268.933)$ & $0.1319(217.708)$ \\
\hline & $T_{(\mathrm{BP})}^{3}$ & $0.0519(\mathbf{3 9 9 . 1 5 3 )}$ & $0.0919(\mathbf{2 6 8 . 9 9 8})$ & $0.1319(\mathbf{2 1 7 . 7 8 3})$ \\
\hline \multirow{4}{*}{ Strategy IV } & $\bar{y}_{\text {SK }}^{4}$ & $0.1118(174.432)$ & $0.1588(155.681)$ & $0.1988(144.476)$ \\
\hline & $\bar{y}_{S K}^{8}$ & $0.1116(185.646)$ & $0.1516(163.049)$ & $0.1916(149.886)$ \\
\hline & $t_{(\mathrm{BN})}^{4}$ & $0.1118(185.647)$ & $0.1516(163.049)$ & $0.1917(149.887)$ \\
\hline & $T_{\text {(BP) }}^{4}$ & $0.1116(\mathbf{1 8 5 . 7 0 1})$ & $0.1517(\mathbf{1 6 3 . 1 1 4})$ & $0.1915(\mathbf{1 4 9 . 9 6 2 )}$ \\
\hline
\end{tabular}

MSE = mean squared error; PRE = percentage relative efficiency. 


\section{References}

Bhushan S and Naqvi N (2015). Generalized efficient classes of estimators in presence of nonresponse using two auxiliary variables, Journal of Statistics and Management Systems, 18, 573602.

Cochran WG (1977). Sampling Techniques (3rd ed), John Wiley and Sons, New York.

Hansen MH and Hurwitz WN (1946). The problem of non-response in sample surveys, Journal of the American Statistical Association, 41, 517-529.

Khare BB and Sinha RR (2007). Estimation of the ratio of the two populations means using multiauxiliary characters in the presence of non-response. In Statistical Techniques in Life Testing, Reliability, Sampling Theory and Quality Control (Pandey BN ed, pp. 163-171), Narosa Publishing House.

Khare BB and Srivastava S (1993). Estimation of population mean using auxiliary character in presence of non-response, National Academy Science Letters, 16, 111-114.

Khare BB and Srivastava S (1995). Study of conventional and alternative two-phase sampling ratio, product and regression estimators in presence of non-response. In Proceedings of the Indian National Science Academy, 65, 195-203.

Khare BB and Srivastava S (1997). Transformed ratio type estimators for the population mean in the presence of non-response, Communications in Statistics - Theory and Methods, 26, 1779-1791.

Okafor FC and Lee H (2000). Double sampling for ratio and regression estimation with sub-sampling the non-respondents, Survey Methodology, 26, 183-188.

Rao PSRS (1986). Ratio estimation with sub sampling the non-respondents, Survey Methodology, 12, 217-230.

Rao PSRS (1987). Ratio and regression estimates with sub sampling the non-respondents. In Paper presented at a special contributed session of the International Statistical Association Meeting, Sept 2-16, Tokyo, Japan.

Searls DT (1964). The utilization of a known coefficient of variation in the estimation procedure, Journal of the American Statistical Association, 59, 1225-1226.

Singh HP, Kumar S, and Kozak M. (2010). Improved estimation of finite population mean using sub sampling to deal with non-response in two phase sampling scheme, Communications in Statistics - Theory and Methods, 39, 791-802.

Singh HP and Kumar S (2008). A regression approach to the estimation of the finite population mean in the presence of non-response, Australian \& New Zealand Journal of Statistics, 50, 395-408.

Singh HP and Kumar S (2010a). Estimation of mean in presence of non-response using two phase sampling scheme, Statistical Papers, 51, 559-582.

Singh HP and Kumar S (2010b). Improved estimation of population mean under the two phase sampling with sub sampling the non-respondents, Journal of Statistical Planning and Inference, 140, 2536-2550.

Shabbir J and Khan NS (2013). On estimating the finite population mean using two auxiliary variables in two phase sampling in the presence of non-response, Communications in Statistics - Theory and Methods, 42, 4127-4145.

Tabasum R and Khan IA (2004). Double sampling for ratio estimation with non-response, Journal of the Indian Society of Agricultural Statistics, 58, 300-306. 